Sandra Cristina Riascos Erazo sandra.riascos@correounivalle.edu.co Calle 4B No. 36-00, Edificio 124 - Oficina 1027. Facultad de Ciencias de la Administración. Universidad del Valle, Sede San Fernando Cali - Colombia.

Doctora en Ingeniería Informática, Universidad Carlos III de Madrid, España. Profesora

Asociada Tiempo Completo, Facultad de Ciencias de la Administración, Universidad del Valle. Miembro del Grupo de Investigación Gestión y Evaluación de Proyectos, Programas y Políticas, Categoría B Colciencias.

Adriana Aguilera Castro adriana.aguilera@correounivalle.edu.co

Calle 4B No. 36-0o, Edificio 124 - Oficina 3017. Facultad de Ciencias de la Administración. Universidad del Valle, Sede San Fernando Cali - Colombia.

Magíster en Ciencias de la Organización, Universidad del Valle. Profesora Asistente Tiempo Completo, Facultad de Ciencias de la Administración. Miembro del Grupo de Investigación Humanismo y Gestión, Categoría A Colciencias.

Artículo de investigación científica y tecnológica Según Clasificación Colciencias

Fecha de recepción: septiembre 02 de 2011 Fecha de corrección: diciembre 10 de 2011 Fecha de aprobación: diciembre 20 de 2011

\section{Herramientas TIC como apoyo a la gestión del talento humano*}

ICT tools as support fort he management of human talent Outils TIC comme soutien à la gestion du talent humain

\section{Resumen}

La incursión de las Tecnologías de la Información y las Comunicaciones (TIC) en el contexto organizacional ha revolucionado el pensamiento administrativo y gerencial, esto se evidencia en varios aspectos, uno de ellos es la forma como se gestiona el talento humano. Este artículo pretende mostrar los resultados de una investigación relacionada con la evaluación de las TIC utilizadas como apoyo a la gestión del talento humano y su objetivo esencial es caracterizar las principales herramientas TIC que se utilizan en los procesos operativos involucrados en la gestión del talento humano. La investigación se desarrolló en dos fases; primera, identificación de las herramientas TIC que apoyan los procesos de gestión humana, y segunda, determinación del software utilizado para la gestión del talento humano en 60 empresas del sector Industrial, Comercial y de Servicios de la ciudad de Santiago de Cali. Los resultados de la investigación indicaron que existen diversas herramientas que facilitan las actividades del área de gestión del talento humano y que se utilizan en mayor proporción en los sectores comercial y de servicios.

Palabras clave: TIC, procesos de gestión del talento humano, administración.

\footnotetext{
* Este artículo presenta los resultados del proyecto de investigación Modelo para la evaluación de las TIC en los procesos administrativos, financiado por la Facultad de Ciencias de la Administración de la Universidad del Valle. Inició en Noviembre de 2008 y el informe final fue entregado en Febrero de 2010.
} 


\section{ICT tools as support fort he management of human talent}

\section{Herramientas TIC como apoyo a la gestión del talento humano}

\section{Outils TIC comme soutien à la gestion du talent humain}

\section{Herramientas TIC como apoyo a la gestión del talento humano}

\section{ICT tools as support fort he management of human talent}

\section{Résumée}

L'incursion des Technologies de l'Information et les Communications (TIC) dans le contexte organisationnel a révolutionné la pensée administrative et de gestion. Cela est évident sur plusieurs aspects, l'un d'eux étant la façon de gérer le talent humain. Cet article prétend montrer les résultats d'une recherche qui porte sur l'évaluation des TIC utilisées comme soutien à la gestion du talent humain, et son objectif principal est de caractériser les principaux outils TIC utilisés dans les processus opératifs présents dans la gestion du talent humain. La recherche s'est développée en deux phases : la première, $l^{\prime}$ identification des outils TIC qui soutiennent les processus de gestion humaine, et la deuxième, la détermination du software utilisé pour la gestion du talent humain dans 60 entreprises du secteur Industriel, Commercial et de Services de la ville de Cali. Les résultats de la recherche ont indiqué qu'il existe plusieurs outils qui facilitent les activités du département de gestion du talent humain et que ceux-ci sont utilisés principalement dans le secteur commercial et de services.

Mots clef: TIC, processus de gestion du talent humain, gestion. 


\section{Herramientas TIC como apoyo a la gestión del talento humano}

\section{Introducción}

Los cambios que afrontan las empresas en la actualidad conducen a analizar y distinguir diversas estrategias que les permitan optimizar sus procesos organizacionales de tal forma que se puedan considerar como competitivas e innovadoras (Porter y Millar, 2009). Los procesos administrativos han sufrido una metamorfosis en su forma de operación dado que a las organizaciones se les exige eficiencia y eficacia; por tanto, se recurre a emplear cada vez más herramientas y a aprovechar las ventajas que suministran las Tecnologías de la Información y las Comunicaciones (TIC). Entre los procesos administrativos más importantes se encuentra la administración del talento humano, este aspecto ha presentado variaciones relacionadas con su significado y operacionalización en las organizaciones, atravesando por tres etapas importantes como son: a) la etapa de las relaciones industriales (primera mitad del siglo XX), b) la administración de recursos humanos (entre 1950 y 1990 aproximadamente), y c) la gestión del talento humano (desde 1990 aproximadamente hasta nuestros días); la última etapa especialmente, implica un interés relevante dado que se aplica en la era de la información, en ésta, la gestión del talento humano es apoyada por sistemas de información y bases de datos que facilitan la toma de decisiones en este contexto (Chavenato, 2002).

Este artículo presenta los resultados de una investigación sobre las diversas utilidades que el mercado tecnológico proporciona para el mejoramiento de actividades fundamentales en la gestión del talento humano, partiendo del desarrollo del proyecto de investigación Modelo para la evaluación de las TIC en los procesos administrativos; el objetivo general de este proyecto fue: Evaluar la efectividad de las TIC en el ámbito administrativo en las Pequeñas y Medianas Empresas de la ciudad Santiago de Cali; y los objetivos específicos: a) contextualizar las TIC que son susceptibles de ser utilizadas en el sector de las Pequeñas y Medianas Empresas de la Ciudad de Cali objeto de la investigación; b) Establecer la metodología de aplicación del modelo de evaluación de la efectividad de las TIC en las Pequeñas y Medianas Empresas de la Ciudad de Cali; c) Aplicar la metodología de evaluación de la efectividad de las TIC en las PYMES de la Ciudad de Cali; d) Analizar los resultados obtenidos en la evaluación de la efectividad de las TIC en las
PYMES de la ciudad de Cali. Adicionalmente, es importante resaltar los resultados que se presentan en relación con el estudio efectuado a 60 empresas del sector Industrial, Comercial y de Servicios de la Ciudad Santiago de Cali, en el que se evidencia la falta de utilización de las herramientas TIC, especialmente, en el sector industrial; a diferencia de lo ocurre en los sectores comercial y de servicios.

El artículo presenta en su primera parte la metodología empleada para el desarrollo de la investigación, la cual es de carácter cualitativo, categorizada como teórico - descriptiva; en la segunda parte se presentan los aspectos conceptuales relacionados con la gestión del talento humano y las TIC como apoyo administrativo; en la tercera parte se exponen los resultados de la investigación y su respectivo análisis; y finalmente, en la cuarta parte se presentan las conclusiones generales de la investigación.

\section{Metodología}

La metodología empleada indica una investigación de carácter cualitativo, categorizada como teórico - descriptiva, dado que responde a los fundamentos de un aspecto cualquiera de un dominio determinado y provee un cierto conocimiento más o menos preciso sobre un fenómeno dado (Aktouf, 2001, p.39); el estudio se desarrolló en diversas etapas con el propósito de mantener el rigor científico. En este sentido, la investigación pretende la comprensión de un fenómeno desde el interior del mismo, como es la inclusión de las TIC en la dinámica de los procesos administrativos en las organizaciones; complementario a esto es necesario resaltar la determinación del software utilizado para la gestión del talento humano en 60 empresas del sector Industrial, Comercial y de Servicios de la ciudad de Santiago de Cali, lo cual permite identificar la parte objetiva de la temática en estudio. En la investigación, se aplicó el método descriptivo - observacional (Pérez, 2001) con apoyo del método de análisis de contenido, esto se evidencia en la descripción de las utilidades que prestan las diversas herramientas TIC en los procesos de gestión del talento humano. El trabajo de investigación se desarrolló teniendo en cuenta las siguientes etapas: 
Etapa de Contextualización: es importante establecer un marco teórico - conceptual que permita orientar la investigación, por tanto fue importante consultar diversos autores; en gestión del talento humano: Gómez-Mejía y Balkin (2003); Koontz y Weihrich (2004); Sherman, Bohlander y Snell (1999); Chiavenato (2002); Rodríguez (2002); Dessler (2001); De Cenzo y Robbins (2001), Dolan, Valle-Cabrera, Jackson y Schuler (2003); García, Sánchez y Zapata (2008) y en incursión de las TIC en el ambiente administrativo y gerencial: Laudon y Laudon (2008), Sieber, S., Valor, J., Porta, V. (2006), Suarez, A. (2008), entre otros; facilitando de esta manera el análisis de las herramientas TIC que son utilizadas en los procesos operativos de la gestión del talento humano.

Etapa de análisis de los procesos de gestión del talento humano: en esta etapa se realizó una agrupación y análisis de los procesos de gestión del talento humano, que sirviera como base para identificar las herramientas TIC susceptibles de ser utilizadas en cada una de las actividades necesarias para la gestión de las personas en las organizaciones.

Etapa de identificación de herramientas TIC: en esta fase se realizaron las siguientes actividades: a) Búsqueda a nivel de Internet del software de apoyo de gestión de talento humano con mayor difusión a nivel comercial, obteniéndose una muestra de 33 paquetes de software; b) Se analizaron las funcionalidades de cada uno de los software para identificar su aplicabilidad en los diferentes procesos de la gestión del talento humano; c) Complementario al proceso anterior y considerando que existen un total de 2738 PYMES en la ciudad de Cali Yumbo, según datos del Censo Económico 2005, se obtuvo la siguiente muestra:

a. Fórmula del Tamaño

Muestral:

b. Datos para su cálculo

$$
n=\frac{\mathrm{NZ}^{2} \pi(1-\pi)}{\mathrm{Ne}^{2}+\mathrm{Z}^{2} \pi(1-\pi)}
$$

$\mathrm{N}$ (Número total de pequeñas

2738

y medianas empresas de

la ciudad de Cali)

Nivel de confianza

Z

0.94

1.560

0.1

0.5

p

$1-p$

0.5

a. Resultado:

$\mathrm{n}=59.52$ PYMES a evaluar
Se indagó en 60 empresas del sector Industrial, Comercial y de Servicios en la ciudad de Santiago de Cali, el software que utilizan para apoyar la ejecución de los procesos de gestión del talento humano.

Etapa de socialización de resultados: Se compartieron los resultados con personas encargadas del área de personal en diversas organizaciones y se recibió su retroalimentación para futuros proyectos de investigación.

\section{Aspectos conceptuales}

\subsection{El concepto de gestión del talento humano}

La gestión del talento humano ha sido estudiada y definida por diversos autores en las distintas etapas de su desarrollo, a continuación se menciona la definición de gestión humana usada como referente dentro de la investigación que dio origen a este artículo; García, Sánchez y Zapata (2008, p.16) definen la gestión humana como: "la actividad estratégica de apoyo y soporte a la dirección, compuesta por un conjunto de políticas, planes, programas y actividades, con el objeto de obtener, formar, motivar, retribuir y desarrollar al personal requerido para generar y potencializar, el management, la cultura organizacional y el capital social, donde se equilibran los diferentes intereses que convergen en la organización para lograr los objetivos de manera efectiva". La importancia del área se centra en posibilitar la consecución de los intereses personales de los empleados y de los intereses de las organizaciones, por supuesto.

Algunos autores realizan clasificaciones de los procesos relacionados con las políticas y prácticas necesarias para integrar el personal y administrar su trabajo y desarrollo dentro de las organizaciones, entre ellos están: GómezMejía y Balkin (2003); Koontz y Weihrich (2004); Chiavenato (2002); Rodríguez (2002); De Cenzo y Robbins (2001), Dolan, Valle-Cabrera, Jackson y Schuler (2003); García, Sánchez y Zapata (2008). Basándose en la información obtenida a través de la lectura de diferentes textos, se organizó una agrupación conveniente para el desarrollo metodológico y práctico de la investigación (figura 1). La delimitación está relacionada con las funciones del área de gestión del talento humano y con cada uno de los procesos necesarios para el ingreso, mantenimiento y desarrollo de las personas dentro de las organizaciones. 
Figura 1. Los Procesos de Gestión Humana

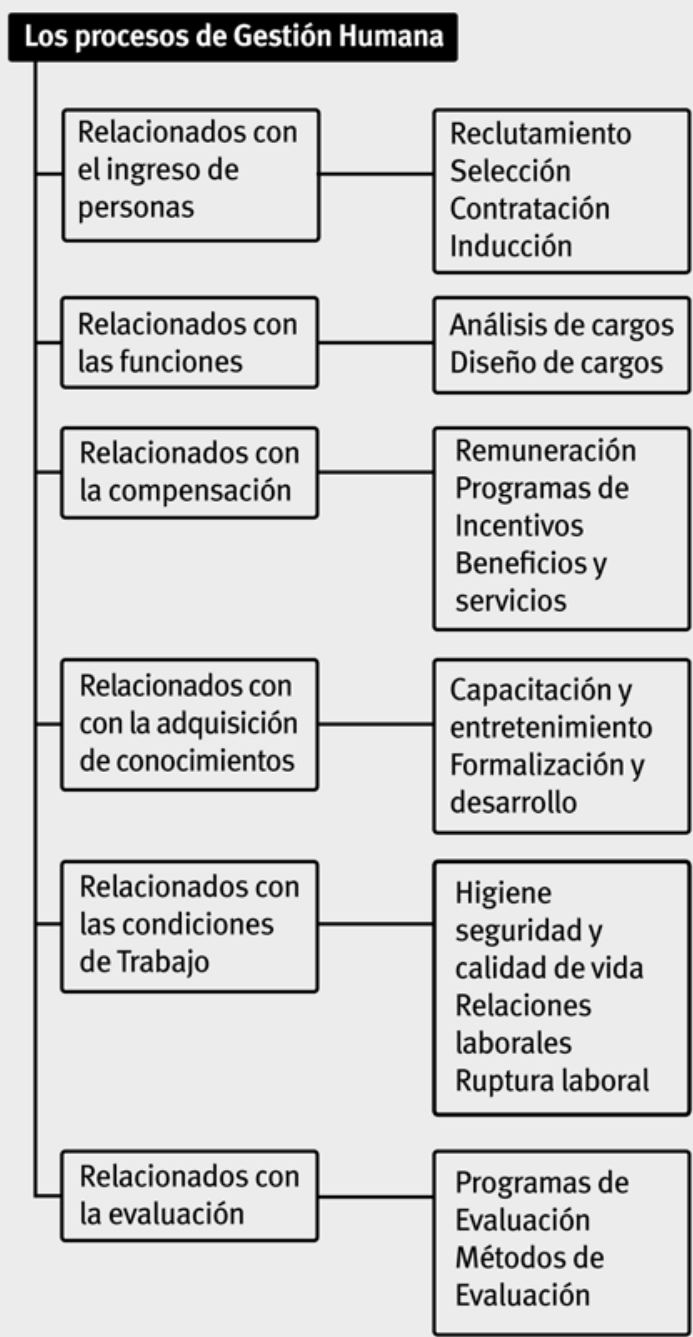

Fuente: Aguielra (2006).

\subsection{Las TIC como apoyo administrativo}

Según Chiavenato (2002) las organizaciones excelentes no son las que tienen la tecnología más avanzada y sofisticada, sino aquellas que saben extraer el máximo provecho a sus tecnologías actuales, es decir aquellas empresas que han identificado las ventajas que implica la correcta utilización de las tecnologías y su efectiva inclusión dentro del desarrollo de los procesos administrativos más importantes de la organización. Precisamente Calderón, Álvarez y Naranjo (2006, p.227) enfatizan "el aporte de la gestión humana en el nuevo modelo tecnoeconómico, que privilegia el conocimiento y las personas como fuente de ventaja competitiva sostenida". Las TIC constituyen la base de las tecnologías que permiten mejorar el manejo de la información, por tanto se convierten en un recurso estratégico, y del uso que se haga del mismo pueden obtenerse ventajas competitivas sostenidas para la empresa (De Pablos, López-Hermoso, Martin-Romo, Medina, Montero, y Nájera, 2006). Según Aguilera y Riascos $(2009$, pp.131,132) "la tecnología ha avanzado considerablemente en función de apoyar esta mutación administrativa..." cuyo principal propósito es el éxito de las organizaciones. Es importante resaltar que aunque las TIC representan una alternativa importante para el progreso de una organización, también se requiere de una planificación y direccionamiento que les permitan su inclusión en los diversos procesos administrativos de forma adecuada. Entre las principales herramientas TIC que se utilizan a nivel administrativo se encuentran las bases de datos y los sistemas de información, estos últimos han sido adoptados en la gran mayoría de los procesos administrativos, las empresas actuales consideran a las TIC y a los sistemas de información en especial, como herramientas de vital importancia en el proceso de conseguir sus objetivos y fundamentalmente en el proceso de constituirse en organizaciones competitivas e innovadoras (Porter y Millar, 2009).

La gestión del talento humano requiere el manejo eficiente de gran cantidad de información, especialmente porque la toma de decisiones en esta actividad es fundamental para el logro de los objetivos organizacionales. Según Chiavenato (2002) la gestión del talento humano requiere la utilización de diversas bases de datos interconectadas que permitan obtener y almacenar datos de diferentes niveles de complejidad, entre las bases de datos más importantes que se requieren están: registro de personal, registro de cargos, registro de secciones, registro de remuneración, registro de beneficios, registro de entrenamiento, registros de candidatos, registros médicos y otros que requiera la organización (figura 2).

Los sistemas de información en la gestión del talento humano facilitan reunir, registrar, almacenar, analizar y recuperar los datos relacionados con los recursos humanos de la organización; los procesos principales que deben ser cubiertos por un sistema de información de gestión del talento humano son: planeación estratégica; formulación de objetivos y programas de acción; registros y controles de personal; registro de nómina; informes sobre remuneraciones; incentivos salariales; beneficios; reclutamiento y selección; entre otros. 
Figura 2. Banco de Datos para un Sistema de Información

\begin{tabular}{|c|c|c|}
\hline Entradas & Banco de datos & Salidas \\
\hline Inclusiones en el registro & Regsitro del personal & Nominas \\
\hline Exclusiones del registro & Registro de cargas & Informes contables \\
\hline Alteraciones del registro & Registro de secciones & Informes legales \\
\hline \multirow{2}{*}{$\begin{array}{l}\text { Tarjetas de registro de } \\
\text { asistencias }\end{array}$} & Registro de remuneración & Reportes de ausentismo \\
\hline & Registro de Beneficios & Reportes de vacaciones \\
\hline Vales y adelantos & Registro de Entrenamiento & Otras informaciones \\
\hline Otras entradas & & \\
\hline
\end{tabular}

Sieber, Valor y Porta (2006) comentan que la necesidad de la interoperabilidad de diferentes sistemas se pone también de manifiesto en el área gestión del talento humano, que al contratar personal para la empresa, se deben determinar en primer lugar, las necesidades de personal, los perfiles de los candidatos, sus tareas y la remuneración que la empresa está dispuesta a ofrecerles. Es importante mencionar que las empresas han asumido el reto de manejar todos sus procesos organizacionales de manera integrada aprovechando los sistemas de información ERP (Enterprise Resource Planning) que facilitan el desarrollo de las actividades administrativas.

Los ERP incluyen los procesos de negocio necesarios para controlar y gestionar las necesidades de recursos humanos de la empresa (gestión de candidatos, elaboración de nóminas, desarrollo de personal, etc.). Los datos están disponibles de forma inmediata para cualquier otra aplicación relacionada, simplificando, por ejemplo, la contabilidad de nóminas. Los ERP están conformados usualmente por los módulos: PM (Personnel Management, Gestión de personal), para administración del personal de la empresa; OM (Organizational Management, Gestión de la organización), para el control de la estructura de la empresa; PA (Payroll Accounting, Gestión de nóminas); TM (Time Management, Gestión del tiempo), para el seguimiento y análisis de los tiempos de los empleados; PD (Personnel Development, Desarrollo personal), asiste en la planificación, monitorización y análisis de seminarios, cursos de formación y reservas de alojamiento, etc. (Sieber, Valor y Porta, 2006).

Entre los ERP de mayor difusión en el ambiente empresarial encontramos SAP (software propietario) y Openerp (software libre) según el estudio realizado por Gartner, Inc. (2008); en esencia los dos incluyen módulos que permiten la gestión del talento humano, SAP está dividido por módulos que realizan distintas funciones y que se integran entre sí para ofrecer compatibilidad en los diferentes procesos de una empresa, estos procesos se pueden agrupar en tres áreas: financiera, logística y de recursos humanos. El área de recursos humanos: incluye los procesos necesarios para la gestión del talento humano; abarca componentes para la administración de salarios y nóminas, contratación de personal, desarrollo y formación, gestión de remuneración, gestión de viajes, beneficios y control de costos de personal. La integración es una de las principales características del SAP, pues la información es compartida en todos los módulos, lo que permite conocer en tiempo real las necesidades y el diagnóstico de lo que se quiere saber; de igual modo, la información se encuentra en línea y existe una jerarquía de la misma (Suárez, 2008).

Antes de presentar los resultados de la investigación, es interesante mencionar como lo plantea Calderón (2006, p. 23) que se espera que "los sistemas de gestión humana no sólo lideren procesos de cambio, sino que desarrollen la capacidad de cambio (flexibilidad, adaptabilidad, agilidad e innovación de la organización)"; en este sentido el apoyo de herramientas TIC juega un papel fundamental para lograr el mejoramiento de los resultados operativos y estratégicos y por consiguiente la eficacia de la empresa.

\section{Resultados de la investigación y discusión}

Los resultados de la investigación se pueden dividir en dos partes: la primera, en relación con la indagación de las herramientas TIC que se pueden utilizar en los diferentes procesos que involucra la gestión del talento humano (Figura 1); la segunda, presenta la identificación del software utilizado en 
60 empresas del sector industrial, comercial y de servicios como apoyo al área de gestión del talento humano.

\subsection{Identificación de herramientas TIC utilizadas en los procesos de Gestión del Talento Humano}

A continuación se relación las herramientas TIC especializadas que pueden ser utilizadas en cada unos de los procesos de Gestión del Talento Humano.

4.1.1. Relacionados con el Ingreso de PersonasEl profesional actual encargado de las funciones de reclutamiento, selección, contratación e inducción se apoya en herramientas TIC como las ilustradas en la figura 3:

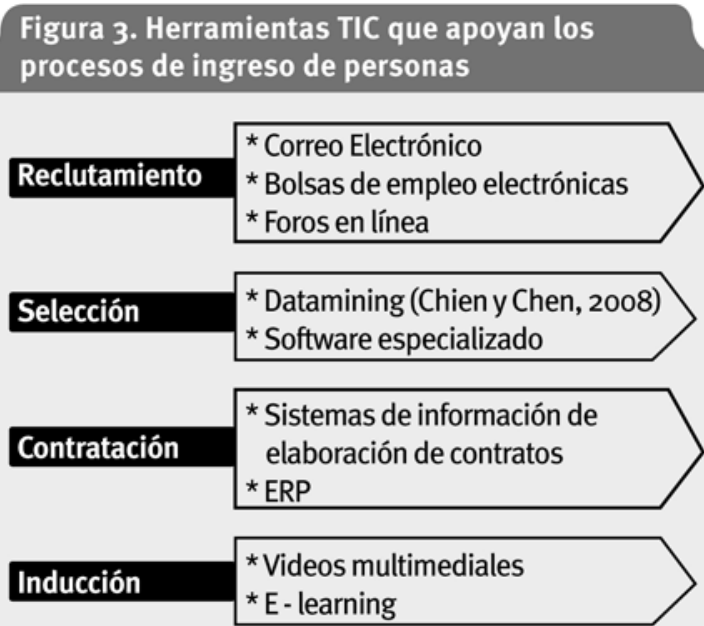

Fuente: elaboración propia.

El reclutamiento es el "proceso de generar un conjunto de candidatos cualificados para un puesto en particular" (Gómez-Mejía y Balkin, 2003, p.250) a nivel general, el reclutamiento se apoya en herramientas TIC como el correo electrónico, las bolsas de empleo electrónicas, dado que el trabajador de la era de la economía del conocimiento, incluye su curriculum vitae en los sitios web destinados a la promoción y divulgación de los profesionales con mayores competencias laborales; otro mecanismo tecnológico que se utiliza hoy en día para el reclutamiento son los foros en línea en los cuales existe la posibilidad de reclutar profesionales de alto nivel. La selección es "el proceso para elegir entre varios candidatos, de dentro o fuera de la organización, a la persona más indicada para ocupar un puesto en ese momento o en el futuro" (Koontz y Weihrich, 2004, p.381); Chien y Chen (2008) comentan que es de vital importancia para las empresas industriales de alta tecnología contar con personal calificado $y$, por tanto, encontraron que la actividad de selección de personal debería ser apoyada por mecanismos tecnológicos como el Datamining; su investigación muestra como esta estrategia fundamentada en un árbol de decisión y reglas asociadas, genera reglas útiles para la selección de personal. Otro paquete computacional especializado que facilita la selección de personal es Spring, este contribuye a racionalizar los procesos de selección y reduce las tareas administrativas, costos y escalas de tiempo.

La investigación en su primera fase de identificación de software comercial evidenció que buena parte del software identificado tiene funciones que apoyan los procesos de reclutamiento y selección (42,4\% y 48,4\% respectivamente) (cuadro 1). En cuanto a la contratación, en el artículo 23 del Código Sustantivo del Trabajo se establece que para que exista contrato de trabajo se requiere que concurran tres elementos esenciales: la actividad personal del trabajador, la continuada subordinación o dependencia del trabajador respecto del patrono, y un salario como retribución del servicio; y por último, la inducción "implica dotar a los nuevos empleados de información preliminar sobre la empresa, sus funciones, sus tareas y su personal" (Koontz y Weihrich, 2004, p.396); para la contratación y la inducción no se encontraron funciones de apoyo en la muestra del software identificado (Cuadro 1).

\begin{tabular}{|c|c|c|c|c|}
\hline Nombre Software & 1 & 2 & 3 & 4 \\
\hline UNICLASS - Nómina y recursos humanos & & $\mathrm{x}$ & $\mathrm{x}$ & $\mathrm{x}$ \\
\hline GOSEM GESTIÓN HUMANA & & $\mathrm{x}$ & & $\mathrm{x}$ \\
\hline Queryx*SRH & $x$ & $\mathrm{x}$ & $x$ & \\
\hline ZUE- sistema de recurso humano & $x$ & $\mathrm{x}$ & $x$ & \\
\hline SAPHCM - HUMAN CAPITAL MANAGEMENT & $x$ & $\mathrm{x}$ & $\mathrm{x}$ & \\
\hline TLM NOMUS & & & $\mathrm{x}$ & \\
\hline ADAMS & $x$ & $\mathrm{x}$ & $\mathrm{x}$ & \\
\hline HEINSOHN HGSS.A. & $\mathrm{x}$ & $\mathrm{x}$ & & \\
\hline KACTUS-HR & $\mathrm{x}$ & $\mathrm{x}$ & & \\
\hline Microsoft Dynamics AX & $x$ & $\mathrm{x}$ & & \\
\hline MIDASOFT & $\mathrm{x}$ & $\mathrm{x}$ & & \\
\hline SARA & $\mathrm{x}$ & $\mathrm{x}$ & & \\
\hline COLSIN-RH & $\mathrm{x}$ & $\mathrm{x}$ & & \\
\hline ICEBERG TH & & $\mathrm{x}$ & & \\
\hline PeopleNet 7 & & $\mathrm{x}$ & & \\
\hline STONE HRM - Human Resource Management & & $\mathrm{x}$ & & \\
\hline SEREX-IBESLSV & $\mathrm{x}$ & & & \\
\hline SISTEMAUNO - NM & $\mathrm{x}$ & & & \\
\hline SOFTLAND SOLUCIÓN CORPORATIVA- NÓMINA & $x$ & & & \\
\hline Visual T.N.S. & $\mathrm{x}$ & & & \\
\hline
\end{tabular}


4.1.2. Relacionados con las Funciones: Como apoyo a la ejecución de las actividades de análisis de cargos y diseño de cargos, las TIC proporcionan las siguientes herramientas que pueden ser utilizadas por el área de gestión del talento humano como lo muestra la figura 4.

Figura 4. Herramientas TIC utilizadas en los procesos relacionados con las funciones

\begin{tabular}{|c|c|}
\hline Análisis de puestos & Diseño de Cargos \\
\hline ERP & $\begin{array}{c}\text { Workflow } \\
\text { Software específico }\end{array}$ \\
\hline $\begin{array}{c}\text { Herramientas de } \\
\text { simulación }\end{array}$ \\
\hline
\end{tabular}

Fuente: Elaboración propia.

El análisis de cargos "es el procedimiento para establecer las obligaciones y las habilidades que requiere un puesto y el tipo de persona que se debe contratar para ocuparlo" (Dessler, 2001, p.84); y el diseño de cargos "incluye la especificación del contenido de cada cargo, los métodos de trabajo y las relaciones con los demás cargos" (Chiavenato 2002, p.167).

Los ERP representan un gran apoyo; el caso de SAP con su módulo de RR.HH, en este se evalúan las necesidades de personal que la empresa requiere, se seleccionan los candidatos y, posteriormente, se contratan. De igual forma, a través del módulo RR.HH se integran los procesos relacionados con la creación de las vacantes y sus publicaciones; estos dos aspectos contemplan la formación del individuo para el cargo disponible bajo los requerimientos que se solicitan, y la forma en la que se publicará la vacante; es decir, el medio con el que se dará a conocer tanto interna como externamente el puesto a ocupar (prensa, televisión, Internet, Intranet, bolsas de empleo y empresas de servicio temporal, entre otras) (Suárez, 2008). Otras herramientas que apoyan el proceso de diseño de cargos son los flujos de trabajo (Workflow) a través de herramientas computarizadas como DFD (Data Flow Diagram), DIA, Microsoft Vision, entre otros; así como también la simulación de cargos a través de algún software como Oragnhrm y Uniclass.

En cuanto a software específico, dentro del proceso investigativo se encontró que de la muestra de los 33 paquetes de software el $18,1 \%$ tienen funciones que permiten apoyar las actividades de análisis y el $18,1 \%$ actividades de diseño de car- gos como lo ilustra el cuadro 2; esto quiere decir, que para las actividades de análisis y diseño de cargos existe un bajo porcentaje de software de apoyo (Cuadro 2).

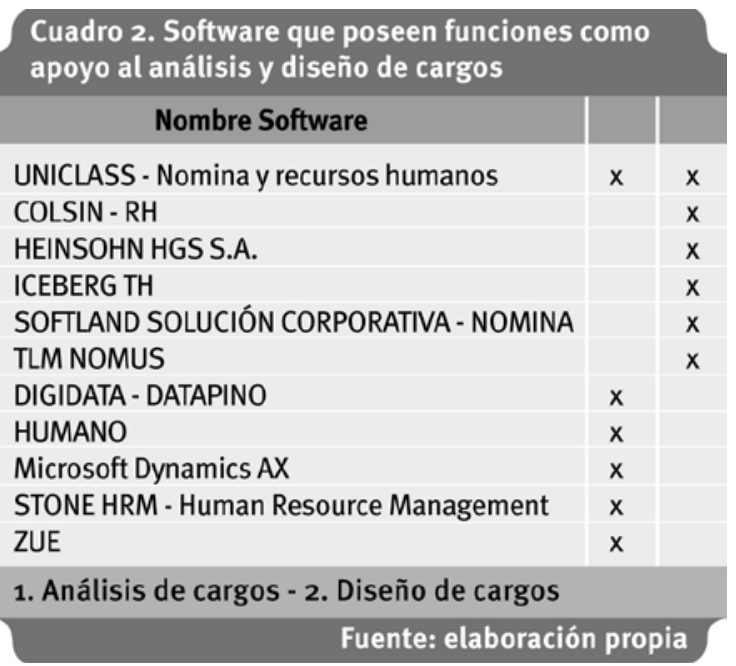

4.1.3 Relacionados con la Compensación: Las actividades relacionadas con el proceso de compensación pueden ser mejoradas a través de herramientas TIC como se indica en la figura 5.

Figura 5. Herramientas TIC utilizadas en

procesos relacionados con la compensación

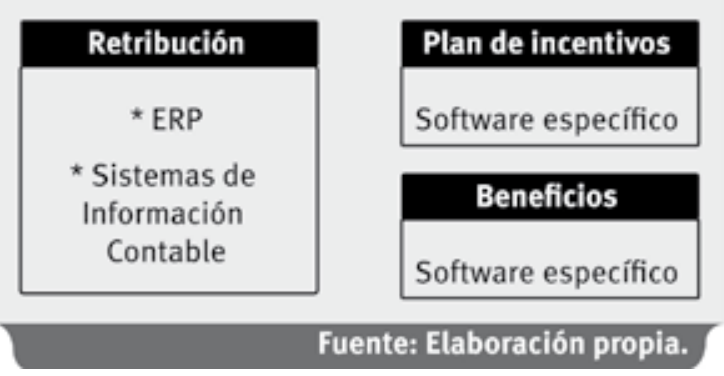

Según Gómez-Mejía, et al (2003), la retribución, de manera general, es el conjunto de las recompensas cuantificables que recibe un empleado por su trabajo. Las empresas manejan varios tipos de programas de incentivos; beneficios adicionales relacionados con la productividad. Los beneficios o prestaciones "las prestaciones son recompensas colectivas que proporcionan seguridad a los empleados y a los miembros de sus familias" (Gómez-Mejía, Balkin y Cardy, 2001, p.398).

Los pagos se hacen cada día más eficientes y efectivos, como lo plantea Rodríguez (2007) al comentar que los pagos de aportes se pueden realizar vía electrónica, utilizando el sistema PILA (Planilla Integrada de Liquidación de Aportes), 
este nuevo sistema permite que los usuarios realicen el pago integrado de los aportes a la seguridad social y demás parafiscales, a través de transferencias electrónicas de fondos.

De acuerdo con la identificación sobre software específico, se observa que todos $(100 \%)$ los paquetes analizados manejan el proceso de retribución pero sólo el $9 \%$ tienen la función de plan de incentivos y únicamente $27,2 \%$ tienen la función de beneficios (cuadro 3). Lo anterior evidencia que las actividades relacionadas con la compensación son un requerimiento funcional imprescindible para el desarrollo de software que apoya las funciones del área de gestión del talento humano (Cuadro 3).

\begin{tabular}{|c|c|c|c|}
\hline Nombre Software & 1 & 2 & 3 \\
\hline COLSIN-RH & $\mathrm{x}$ & $\mathrm{x}$ & $\mathrm{x}$ \\
\hline Amazing HCM PayRoll & $\mathrm{x}$ & $\mathrm{x}$ & $\mathrm{x}$ \\
\hline KACTUS-HR & $\mathrm{x}$ & $\mathrm{x}$ & $\mathrm{x}$ \\
\hline ICEBERG TH & $\mathrm{x}$ & & $\mathrm{x}$ \\
\hline TLMNOMUS & $\mathrm{x}$ & & $\mathrm{x}$ \\
\hline DIGIDATA - DATAPINO & $\mathrm{x}$ & & $\mathrm{x}$ \\
\hline HUMANO & $\mathrm{x}$ & & $\mathrm{x}$ \\
\hline ADMAS & $\mathrm{x}$ & & $\mathrm{x}$ \\
\hline Visual T.N.S. & $\mathrm{x}$ & & $\mathrm{x}$ \\
\hline UNICLASS - Nómina y recursos Humanos & $\mathrm{x}$ & & \\
\hline HEINSOHN HGSS.A. & $\mathrm{x}$ & & \\
\hline SOFTLAND SOLUCIÓN CORPORATIVA & $\mathrm{x}$ & & \\
\hline Microsoft Dynamics AX & $\mathrm{x}$ & & \\
\hline STONE HRM - Human Resource Management & $\mathrm{x}$ & & \\
\hline ZUE & $\mathrm{x}$ & & \\
\hline FACTORYVISUAL & $\mathrm{x}$ & & \\
\hline FOMPLUS.net & $\mathrm{x}$ & & \\
\hline GOSEM GESTIÓN HUMANA & $\hat{x}$ & & \\
\hline HELISAGW & $\mathrm{x}$ & & \\
\hline Ilimitada NOMINAY AUTOLIQUIDACIONES (RHI) & $\hat{x}$ & & \\
\hline LOGISYS & $\hat{x}$ & & \\
\hline MIDASOFT & $\hat{x}$ & & \\
\hline NGSoft Nómina & $\mathrm{x}$ & & \\
\hline NOVASOFT & $\hat{x}$ & & \\
\hline OFIMATICA & $\hat{x}$ & & \\
\hline PeopleNet 7 & $\mathrm{x}$ & & \\
\hline Queryx*SRH & $\hat{x}$ & & \\
\hline SAPHCM - HUMAN CAPITAL MANAGEMENT & $\mathrm{x}$ & & \\
\hline SARA SEREX-IBESLSV & $\mathrm{x}$ & & \\
\hline SISTEM UNO - NM & $\mathrm{x}$ & & \\
\hline SOFILAND - Recursos Humanos \& Nómina & $\mathrm{x}$ & & \\
\hline ZUE-Sistema de Recursos Humanos & $x$ & & \\
\hline
\end{tabular}

4.1.4. Relacionados con la Adquisición de Conocimientos: El mejoramiento de este proceso es fundamental para la gestión del talento humano, por eso las TIC suministran herramientas que apoyan estas actividades (figura 6).
Figura 6. Herramientas TIC como apoyo a

procesos de adquisición de conocimientos

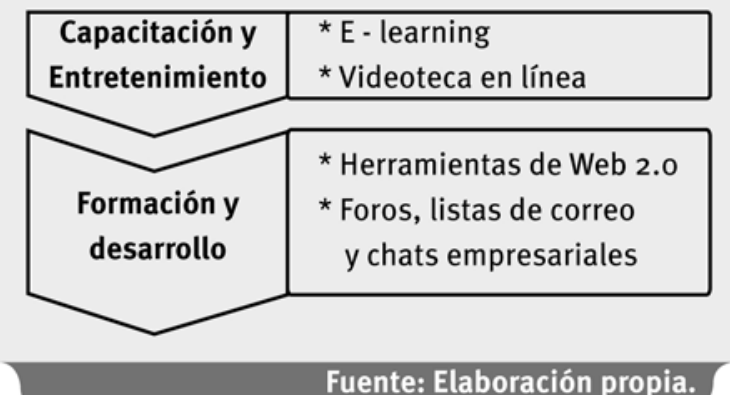

149

En cuanto a la capacitación y entrenamiento se puede decir que "la capacitación de personal es un proceso que se relaciona con el mejoramiento y crecimiento de las aptitudes de los individuos y de los grupos dentro de la organización. La meta de la capacitación de personal es facilitar el logro de metas organizacionales" (Rue y Byars, 2000, p.157). Y por otro lado "El entrenamiento es un proceso que comprende la adquisición de destrezas, conceptos, reglas o actitudes. La meta del entrenamiento es mejorar el rendimiento actual y futuro" (Rue y Byars, 2000, p.349). En cuanto a la formación y desarrollo del empleado, "La formación trata de proporcionar al empleado habilidades específicas o corregir deficiencias en su rendimiento" (Dolan, et al, 2003, p.119) y "el desarrollo es un esfuerzo para ofrecer a los empleados las habilidades que la organización necesitará en el futuro" (Gómez-Mejía, et al, 2001, p.283).

Entre las TIC que facilitan el desarrollo de actividades relacionadas con la adquisición de conocimientos están el E-Learning que es una forma de educación a distancia basada en medios electrónicos, es decir los cursos virtuales que le ayudan al empleado a capacitarse constantemente; la videoteca en línea que proporciona la red Internet a través de diversos sitios como por ejemplo: Youtube.com, video.google.com; herramientas Web 2.0 que son la transición que se ha dado de aplicaciones tradicionales hacia aplicaciones que funcionan a través de la web enfocadas al usuario final, como por ejemplo: googledocs y googlegroups; foros, listas de correo y chats empresariales, son herramientas virtuales que le facilitan al empleado establecer relaciones con personas que tienen su mismo cargo en otras organizaciones y aprender de sus experiencias, entre los más conocidos están: gestionhumana.com, gestionhumanaforoactivo. net, vcgestionhumana.com, entre otros. 
A nivel de software específico de acuerdo con la identificación, el 30,3\% de paquetes apoyan las actividades de capacitación y $24,2 \%$ las de formación y desarrollo como se ilustran en el cuadro 4.

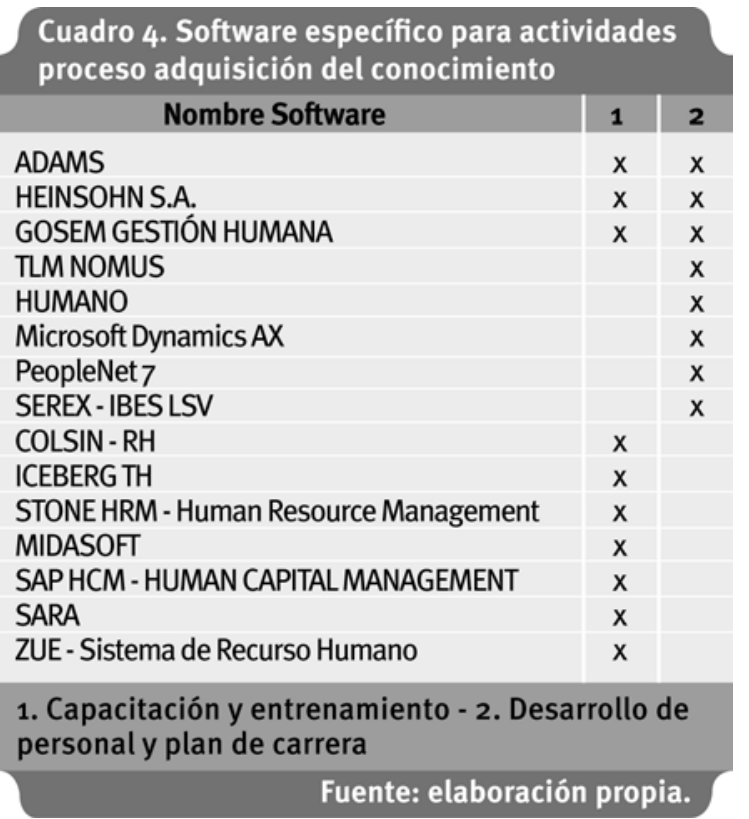

\subsubsection{Relacionados con las Condiciones} de Trabajo: Las actividades que involucra el proceso relacionado con las condiciones de trabajo se apoyan con la utilización de herramientas TIC como las señaladas en la figura 7.

Figura 7. Herramientas TIC utilizadas en procesos relacionados con condiciones de trabajo

\section{Higiene}

Software de diagnóstico - Sensores de atomatizados

\section{Seguridad}

Videos interactivos - Avisos multimediales

\section{Calidad de vida laboral}

Herramientas WEB - Divulgación de eventos en línea

\section{Relaciones laborales \\ Foros y chat - Grupos en línea}

\section{Ruptura laboral}

Correo electrónico - Bases de datos

\section{Fuente: elaboración propia.}

Según Rodríguez (2002) la higiene se entiende como las condiciones o prácticas que conducen a un estado de buena salud y la seguridad se entiende como las acciones o prácticas que conducen a evitar accidentes.
Según Chiavenato citado por García (2008, p.49) la calidad de vida laboral "representa el grado de satisfacción de las necesidades personales de los miembros de la organización mediante el trabajo".

Entre las herramientas TIC que apoyan los procesos relacionados con las condiciones de trabajo se distinguen: el software de diagnóstico, es decir paquetes que facilitan determinar las condiciones laborales que ofrece la organización, como por ejemplo el software ISOSYSTEM que facilita gestionar y monitorear el sistema de salud y seguridad laboral; además de otras herramientas como la instalación de sensores automatizados que le permitan al personal contar con un espacio apropiado para el desarrollo de sus labores; los videos interactivos y los avisos multimediales son de gran ayuda para los procesos de seguridad del personal; la calidad de vida laboral se puede apoyar en herramientas web como la agenda o el calendario que le permita publicar los eventos culturales de la organización, así como también la página web de la organización para mantener un nivel de comunicación adecuado.

A nivel general dentro del software específico se encontró que el $39,4 \%$ apoya las actividades de higiene y seguridad industrial (Cuadro 5); en relación con las actividades de calidad de vida no se encontraron funcionalidades en el software estudiado que apoye este proceso.

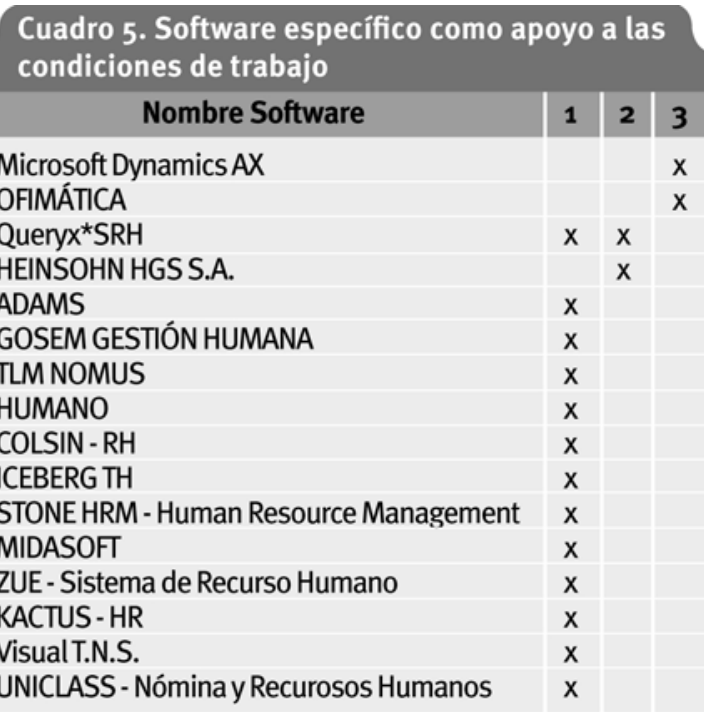

1. Higiene y seguridad industrial - 2. Relaciones laborales - 3. Ruptura laboral

Fuente: elaboración propia. 
4.1.6. Relacionados con la Evaluación: Las actividades que encierran los procesos de evaluación se apoyan en herramientas TIC que les facilitan su ejecución además de permitirles obtener resultados más efectivos. Entre las más utilizadas están las relacionadas en la figura 8.

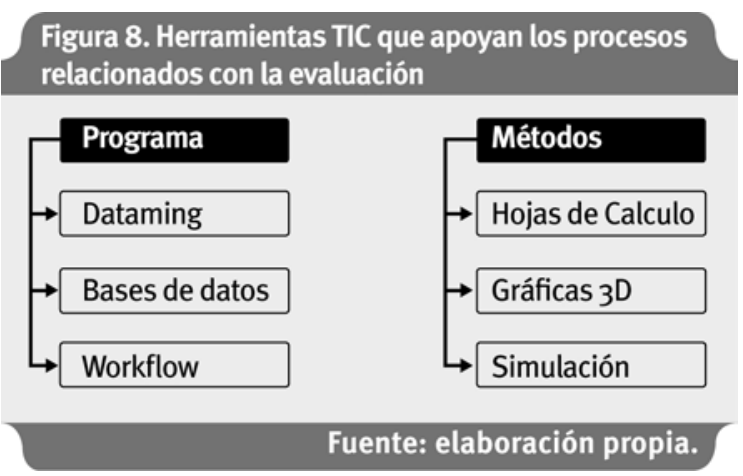

Según Gómez, et al (2001, p.244,245); implementar un programa de evaluación del desempeño exige identificar áreas de trabajo a las cuales hay que medirles el rendimiento porque dependiendo de éste se afecta positiva o negativamente el éxito de una organización, luego debe llevarse a juicio el comportamiento del empleado, y se debe tener muy claro que el objetivo de la evaluación no es generar simples críticas, por el contrario debe enfocarse hacia el futuro, es decir, lo que los empleados pueden hacer en pro de alcanzar su potencial dentro de la organización. Según Rodríguez (2002, p.333), los métodos de evaluación del desempeño son un medio para obtener datos e informaciones que puedan ser registrados y procesados, y utilizarlos para la toma de decisiones y soluciones que traten de mejorar e incrementar el desempeño humano dentro de las organizaciones.

Los procesos relacionados con la evaluación dentro de la gestión talento humano son de vital importancia, por tanto, la escogencia de las herramientas TIC que permiten apoyar dichos procesos deben responder a los criterios de eficacia y eficiencia, en este sentido el Datamining, las Bases de datos y WorkFlow facilitan la identificación de los niveles de efectividad de las diversas áreas de trabajo así como también le proporcionan al área información suficiente para una efectiva toma de decisiones; los métodos de evaluación se apoyan en herramientas como las hojas de cálculo, las gráficas 3 D que ilustran adecuadamente los datos necesarios y la simulación que permite tener una visión prospectiva sobre el funcionamiento de las diversas áreas de trabajo.
En cuanto a software específico se refiere, se evidencia que el $36,36 \%$ apoya los procesos de evaluación de desempeño y el $9 \%$ colaboran en el método de evaluación, esto quiere decir que la incursión de las TIC en los procesos de evaluación es limitada (Cuadro 6).

Cuadro 6. Software especifico como apoyo al proceso de evaluación

Nombre Software \begin{tabular}{l|l}
1 & 2 \\
\hline & $x$
\end{tabular}

SAPHCM-HUMAN CAPITALMANAGEMENT $\quad X \quad X$ ICEBERG TH

DIGIDATA - DATAPINO

Queryx*SRH

GOSEM GESTIÓN HUMANA

HUMANO

COLSIN-RH

STONE HRM - Human Resource Management

MIDASOFT

ZUE-Sistema de Recurso Humano

KACTUS-HR

UNICLASS - Nómiba y Recursos Humanos

PeopleNet 7

SEREX-IBESLSV

1. Desempeño - 2. Evaluación

Fuente: elaboración propia.

\subsection{Software utilizado como apoyo a los procesos de Gestión del Talento Humano}

En esta fase de la investigación se determinó el software que es usado en 60 empresas del sector Industrial, Comercial y de Servicios para efectuar las actividades relacionadas con los procesos de gestión de talento humano; en el cuadro 7 , se presenta un resumen de los paquetes software utilizados en la muestra de empresas.

Los resultados permiten deducir que en el sector industrial existe una alta inutilización $(69 \%)$ de paquetes software que apoyan los procesos de la gestión de talento humano; el $31 \%$ utiliza para este fin las utilidades que suministran los ERP específicamente los módulos de RRHH, como SAP; también se evidencia la utilización de software propio o desarrollos in house de las organizaciones. Considerando que las herramientas TIC son mecanismos que permiten mejorar los procedimientos en las organizaciones, la baja utilización de estas herramientas en los procesos de gestión humana, podría ocasionar inadecuado seguimiento y control del personal en actividades tan importantes como la evaluación del desempeño, los programas de formación, los esquemas de retribución, reclutamiento y selección, análisis y diseño de cargos, entre otros. 
Cuadro 7. Resumen de software existente en las 60 empresas estudiadas

\begin{tabular}{|c|c|c|c|c|c|}
\hline & & \multicolumn{4}{|c|}{ Sector al que pertenece } \\
\hline & & Industria & Comercio & Servicios & Total \\
\hline \multirow{21}{*}{ 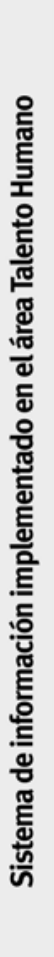 } & 1 Pyme & $100 \%$ & & & $100 \%$ \\
\hline & Autocad & & & $100 \%$ & $100 \%$ \\
\hline & $\mathrm{CG}_{1}$ & $50 \%$ & & $50 \%$ & $100 \%$ \\
\hline & Computador, red, Internet, sistemas contables & $100 \%$ & & & $100 \%$ \\
\hline & Ensa & & & $100 \%$ & $100 \%$ \\
\hline & Helisa & $100 \%$ & & & $100 \%$ \\
\hline & Hware & & & $100 \%$ & $100 \%$ \\
\hline & Ilustrator, photoshop & & & $100 \%$ & $100 \%$ \\
\hline & Orion Software & & & $100 \%$ & $100 \%$ \\
\hline & Paquete Office & $66,7 \%$ & & $33,3 \%$ & $100 \%$ \\
\hline & Red, telefonía fija y móvil, correo electrónico, ordenador & $100 \%$ & & & $100 \%$ \\
\hline & Saiopen & $100 \%$ & & & $100 \%$ \\
\hline & Sistema de Nómina & $50 \%$ & & $50 \%$ & $100 \%$ \\
\hline & Sistema de información & $100 \%$ & & & $100 \%$ \\
\hline & Software propio & $100 \%$ & & & $100 \%$ \\
\hline & Supracont & $100 \%$ & & & $100 \%$ \\
\hline & Visual Cam & $100 \%$ & & & $100 \%$ \\
\hline & Ninguno & $69 \%$ & $6,9 \%$ & $24,1 \%$ & $100 \%$ \\
\hline & Sap & $100 \%$ & & & $100 \%$ \\
\hline & Software Daruma & & $100 \%$ & & $100 \%$ \\
\hline & Total & $65 \%$ & $5 \%$ & $30 \%$ & $100 \%$ \\
\hline
\end{tabular}

En el sector de servicios se presenta alta utilización de software de apoyo (75.9\%), se aprecia que existen diversos paquetes de software, el paquete office ofrece un apoyo importante a las empresas para los procesos administrativos, especialmente los de gestión del talento humano, así como también aquellos software relacionados con procesos de nómina, en este aspecto los paquetes contables son los más utilizados. Es importante considerar que para este sector es fundamental la satisfacción de los usuarios; esto justifica que la gran mayoría de las empresas que ofrecen servicios procuran invertir en tecnología que apoye la adecuada gestión del personal, ya que estos son quienes tienen contacto directo con los clientes.

El sector comercial utiliza en gran medida software de apoyo para los procesos de gestión del talento humano (93.1\%). En este sector el software de mayor utilización es el Sistema DARUMA, una solución que apoya de manera integral la Administración, Control y Mejoramiento de los Sistemas de Gestión, permitiendo cumplir con los estándares nacionales e internacionales; en cuanto a la administración del talento humano, se destacan el manejo de indicadores de gestión, administración del riesgo, herramientas de mejoramiento, administración de registros. Este sector se afecta de manera directa por procesos como la globalización del mercado, las economías de escala, la mundialización de la economía; por ello es imperativo que su nivel de innovación en procesos administrativos, como el de gestión del talento humano, involucre inversión en las herramientas tecnológicas que el mercado ofrece.

\section{7. conclusiones}

Aunque existe un número considerable de software que apoya los procesos de gestión del talento humano, no todos conciben funcionalidades para las actividades de esta área. De acuerdo con la investigación, la función que se encuentra en el software identificado en el mercado, se relaciona con el proceso de compensación, específicamente con el manejo de nómina. Considerando que el software ha sido diseñado de acuerdo con los requerimientos de las organizaciones, se puede deducir que estas se preocupan principalmente por establecer esquemas de control en la remuneración de los empleados y esto puede permitir avanzar hacia procesos de equidad interna y externa. 
La incursión de los servicios de internet en el contexto de las actividades operativas de las organizaciones se evidenció en la investigación mediante la utilización de las bolsas de empleo electrónicas, correo electrónico, páginas web, foros, chat, entre otros, especialmente útiles para el funcionamiento del área de gestión del talento humano, brindando agilidad en los procesos de comunicación, reclutamiento, formación y evaluación de desempeño; por tanto cabe mencionar que los recursos de internet han disminuido notoriamente la inversión en tecnología in house. Además con la implementación de servicios oursourcing se favorecen las estrategias de innovación que pretenden implementar las organizaciones y se logra una interesante transformación de los procesos relacionados con la captación de capital humano.

Es indudable el avance en el desarrollo de técnicas TIC como son las redes neuronales, la lógica difusa, algoritmos genéticos, datamining, entre otras; específicamente la técnica del datamining ha sido probada como herramienta eficaz en el apoyo de la ejecución de procesos de selección de personal y evaluación del desempeño que forman parte de la gestión del talento humano. Esto contribuye al mejoramiento de la calidad de los productos $y / 0$ servicios que ofrecen las organizaciones.

La diversidad de paquetes de software útiles para apoyar procesos de Gestión del Talento Humano, comprueba que estos se han convertido en un producto necesario para la industria de las TIC, debido al incremento de su uso por parte de todo tipo de organizaciones, especialmente en el sector comercial y de servicios.

La clasificación de los procesos de Gestión del Talento Humano y la documentación de las herramientas TIC que los apoyan, hacen de este artículo una muy buena fuente de consulta para las personas interesadas en el mejoramiento de los procesos de gestión del talento humano en las organizaciones.

\section{Referencias}

Aguilera, A (2006). Los Procesos de Gestión Humana y la Estrategia Organizacional. Berlín, Alemania: Editorial Académica Española.

Aguilera, A. y Riascos S. (2009) Direccionamiento Estratégico apoyado en las TIC. Estudios Gerenciales, 111(25), pp. 131,132 .
Aktouf, 0. (2001). La metodología de las Ciencias Sociales y el Enfoque Cualitativo en las Organizaciones. Una introducción al procedimiento clásico y una crítica. Colombia: Universidad del Valle. Facultad de Ciencias de la Administración.

Calderón, G. (2006). La Gestión Humana y sus aportes a las organizaciones Colombianas. Cuadernos de Administración - Pontificia Universidad Javeriana de Bogotá, 19 (31), p. 23.

Calderón, G. Álvarez y C. Naranjo, J. (2006). Gestión Humana en las Organizaciones Un Fenómeno Complejo: Evolución, Retos, Tendencias y Perspectivas de Investigación. Cuadernos de Administración - Pontificia Universidad Javeriana de Bogotá, 19 (32), p. 227.

Chiavenato, I. (2002). Gestión del talento humano: el nuevo papel de los recursos humanos en las organizaciones. Bogotá, Colombia: McGraw-Hill.

Chien, Ch. y Chen, L. (2008). Data mining to improve personnel selection and enhance human capital: A case study in high-technology industry. Expert Systems with Applications: An International Journal, n. 34, pp. $280-290$.

De-Cenzo, David A. y Robbins, S. (2001). Administración de recursos humanos. México D.F.: Editorial Limusa S.A.

De-Pablos, C., López-Hermoso, J., MartinRomo, S., Medina, S., Montero, A. y Nájera, J. (2006). Dirección y gestión de los sistemas de información en la empresa, una visión integradora. Madrid, España: ESIC.

Dessler, G. (2001). Administración de personal. México D.F.: Prentice Hall Hispanoamericana S.A.

Dolan, S., Valle, R., Jackson, S. y Schuler, R. (2003). La gestión de los recursos humanos. Preparando profesionales para el siglo XXI. Madrid, España: McGraw-Hill.

García, M., Sánchez, K. y Zapata, A. (compiladores). (2008). Perspectivas Teóricas para el estudio de la Gestión Humana. Una relación con el capital social, la cultura organizacional y el management. Cali, Colombia: Programa Editorial Universidad del Valle. 
Gartner Inc. (2008). The Top 50 Global Enterprice Application Vendors. Disponible en http:// www.gartner.com/technology/supply-chain/ top25.jsp?compURI=tcm:7-44652 Consultado en Enero 23 de 2011.

Gómez-Mejía, L. y Balkin, D. (2003). Administración. Madrid, España: McGraw-Hill.

Gómez-Mejía, L., Balkin, D. y Cardy, R. (2001). Dirección y gestión de recursos humanos. Madrid, España: Prentice Hall. Pearson Educación S.A.

Koontz, H. y Weihrich, H. (2004). Administración. Una perspectiva global. México D.F.: McGraw-Hill.

Laudon, K. y Laudon, G. (2008). Sistemas de información gerencial. México D.F.: Pearson Education.

Pérez, G. (coord) (2001). Modelos de Investigación cualitativa. Madrid, España: Narcea.

Porter, M. y Millar, V. (2009). Cómo obtener ventaja competitiva por medio de la información. Barcelona, España: Deusto
Rodríguez, C. A. (2007). Conozca todo sobre el pago de aportes por vía electrónica. Recuperado el 27 de septiembre del 2009, http://www.gestionhumana.com/gh4/ BancoConocimiento/P/pila/pila.asp?.

Rodríguez, J. (2002). Administración moderna de personal. México, D.F.: Thomson.

Rue, W. y Byars, L. (2000). Administración: Teoría y Aplicaciones. México D.F.: Alfaomega.

Sherman, A., Bohlander, G. y Snell, S. (1999). Administración de recursos humanos. México D.F.: Thomson Editores.

Sieber, S., Valor, J. y Porta, V. (2006). Los sistemas de información en la empresa actual, aspectos estratégicos y alternativas tácticas. Madrid, España: McGraw-Hill.

Suárez, A. (2008). SAP: Integración y gestión para las organizaciones. Recuperado el 24 de septiembre del 2009, http://www.gestionhumana.com/gh4/BancoConocimiento/S/sap/ sap.asp. 